\title{
A New $k$-th Derivative Estimate for Exponential Sums via Vinogradov's Mean Value
}

\author{
D.R. Heath-Brown \\ Mathematical Institute, Oxford
}

In celebration of the 125 th
anniversary of the birth of
Ivan Matveevich Vinogradov

\section{Introduction}

The familiar van der Corput $k$-th derivative estimate for exponential sums (Titchmarsh [8, Theorems 5.9, 5.11, \& 5.13], for example), may be stated as follows. Let $k \geq 2$ be an integer, and suppose that $f(x):[0, N] \rightarrow \mathbb{R}$ has continuous derivatives of order up to $k$ on $(0, N)$. Suppose further that $0<\lambda_{k} \leq f^{(k)}(x) \leq A \lambda_{k}$ on $(0, N)$. Then

$$
\sum_{n \leq N} e(f(n)) \ll A^{2^{2-k}} N \lambda_{k}^{1 /\left(2^{k}-2\right)}+N^{1-2^{2-k}} \lambda_{k}^{-1 /\left(2^{k}-2\right)},
$$

with an implied constant independent of $k$. One usually chooses $k$ so that the first term dominates, and one often has $A^{2^{2-k}} \ll 1$, so that the bound is merely $O\left(N \lambda_{k}^{1 /\left(2^{k}-2\right)}\right)$. Clearly one can only get a non-trivial bound when $\lambda_{k}<1$. A typical application is the series of estimates

$$
\zeta(\sigma+i t) \ll t^{1 /\left(2^{k}-2\right)} \log t, \quad\left(\sigma=1-\frac{k}{2^{k}-2}, t \geq 2\right)
$$

for $k=2,3, \ldots$. Again the implied constant is independent of $k$.

One can improve on the standard $k$-th derivative bound somewhat. Thus Robert and Sargos [6] show roughly that if $k=4$ then

$$
\sum_{n \leq N} e(f(n)) \ll_{\varepsilon} N^{\varepsilon}\left(N \lambda_{4}^{1 / 13}+\lambda_{4}^{-7 / 13}\right),
$$

for any $\varepsilon>0$. In the corresponding version of (1) one would have a term $N \lambda_{4}^{1 / 14}$ in place of $N \lambda_{4}^{1 / 13}$. Similarly for $k=8$ and 9 , Sargos [7, Theorems $3 \& 4$ ] gives bounds

$$
\sum_{n \leq N} e(f(n)) \ll_{\varepsilon} N^{\varepsilon}\left(N \lambda_{8}^{1 / 204}+\lambda_{8}^{-95 / 204}\right),
$$

and

$$
\sum_{n \leq N} e(f(n)) \ll_{\varepsilon} N^{\varepsilon}\left(N \lambda_{9}^{7 / 2640}+\lambda_{9}^{-1001 / 2640}\right),
$$


respectively. Here the exponents $1 / 204$ and $7 / 2640$ should be compared with the values $1 / 254$ and $1 / 510$ produced by (1).

There are quite different approaches to exponential sums, using estimates for the Vinogradov mean value integral

$$
J_{s, l}(P)=\int_{0}^{1} \ldots \int_{0}^{1}\left|\sum_{n \leq P} e\left(\alpha_{1} n+\ldots+\alpha_{l} n^{l}\right)\right|^{2 s} d \boldsymbol{\alpha},
$$

see Vinogradov [9, 10], and Korobov [3], amongst others. The first of these methods is described by Titchmarsh [8, Chapter 6] for example. The VinogradovKorobov machinery has been used by Ford [2, Theorem 2] to show that

$$
\sum_{N<n \leq 2 N} n^{-i t} \ll N^{1-1 / 134 k^{2}}
$$

for $N^{k} \geq t \geq 2$. (Ford's result is somewhat more precise, and more general.) One may think of this as corresponding very roughly to a bound of the form (1) with first term $N \lambda_{k}^{1 / 134 k^{2}}$.

A slightly refined version of the original method of Vinogradov 9 coupled with new estimates for the Vinogradov mean value integral, leads to distinctly stronger bounds. For example, Wooley [11, Theorem 1.2] gives

$$
J_{s, l}(P) \ll_{\varepsilon, l} P^{2 s-l(l+1) / 2+\varepsilon} \quad(s \geq l(l-1),
$$

and Robert [5, Theorem 10] used this to show that if $k \geq 4$ then

$$
\sum_{n \leq N} e(f(n)) \ll_{A, k, \varepsilon} N^{1+\varepsilon}\left(\lambda_{k}^{1 / 2(k-1)(k-2)}+N^{-1 / 2(k-1)(k-2)}\right)
$$

for $N \geq \lambda_{k}^{-(k-1) /(2 k-3)}$. This is a remarkable improvement on the classical $k$-th derivative estimate. The exponent of $\lambda_{k}$ is better than $1 /\left(2^{k}-2\right)$ for all $k \geq 4$, and decreases quadratically rather than exponentially.

The purpose of this paper is to further refine the original method of Vinogradov [9] and to input the very recent optimal bounds for the Vinogradov mean value integral, due to Wooley [11] (for $l=3$ ), and to Bourgain, Demeter and Guth [1] (for $l \geq 4)$. These theorems show that

$$
J_{s, l}(P) \ll_{\varepsilon, l} P^{2 s-l(l+1) / 2+\varepsilon} \quad\left(s \geq \frac{1}{2} l(l+1), l \geq 1\right),
$$

the cases $l=1$ and $l=2$ being elementary. The range for $s$ is optimal, and it is this feature that represents the dramatic culmination of many previous works over the past 80 years. Unfortunately neither result gives an explicit dependence on $l$ and $s$, nor gives an explicit form for the factor $P^{\varepsilon}$. Results prior to the advent of Wooley's efficient congruencing method had required $s$ to be larger, but had given an explicit dependence on $l$. Thus for example, Ford [2, Theorem 3] implies in particular that

$$
J_{s, l}(P) \ll l^{13 l^{3} / 4} P^{2 s-l(l+1) / 2+l^{2} / 1000} \quad\left(s \geq \frac{5}{2} l^{2}, l \geq 129\right),
$$


in which one has an additional term $l^{2} / 1000$ in the exponent, and more restrictive conditions on $l$ and $s$. An important application of bounds for Weyl sums is to the zero-free region for $\zeta(s)$, as described by Ford. However for this it is crucial to have a suitable dependence on the parameter $l$, so that the new result of Bourgain, Demeter and Guth is not applicable.

Our first result gives a new $k$-th derivative estimate

Theorem 1 Let $k \geq 3$ be an integer, and suppose that $f(x):[0, N] \rightarrow \mathbb{R}$ has continuous derivatives of order up to $k$ on $(0, N)$. Suppose further that

$$
0<\lambda_{k} \leq f^{(k)}(x) \leq A \lambda_{k}, \quad x \in(0, N) .
$$

Then

$$
\sum_{n \leq N} e(f(n)) \ll_{A, k, \varepsilon} N^{1+\varepsilon}\left(\lambda_{k}^{1 / k(k-1)}+N^{-1 / k(k-1)}+N^{-2 / k(k-1)} \lambda_{k}^{-2 / k^{2}(k-1)}\right) .
$$

If one thinks of $N \lambda_{k}^{1 / k(k-1)}$ as being the leading term here, then one needs to compare the exponent $1 / k(k-1)$ with the corresponding exponent $1 /\left(2^{k}-2\right)$ in (11). These agree for $k=3$, but for larger values of $k$ the new exponent tends to zero far more slowly than the old one. It may perhaps be something of a surprise that an analysis via Vinogradov's mean value integral reproduces the same term $N \lambda_{3}^{1 / 6}$ as in the classical third-derivative estimate.

We should emphasize that the stength of Theorem 1 comes almost entirely from the new bound (4). One could have injected (4) into the method of Robert [5], to produce an estimate with the same terms $\lambda_{k}^{1 / k(k-1)}+N^{-1 / k(k-1)}$ as in Theorem 1, but valid only for $N \geq \lambda_{k}^{-(k-1) /(2 k-3)}$. Our result, incorporating a slightly better way of using the Vinogradov mean value, gives the terms $\lambda_{k}^{1 / k(k-1)}+N^{-1 / k(k-1)}$ in the substantially longer range $N \geq \lambda_{k}^{-2 / k}$. However for our application to Theorems 25 below, Robert's range would have been very nearly sufficient.

The secondary terms in the bound given by Theorem 1 are somewhat awkward. The classical estimate (11) leads easily to an exponent pair,

$$
\left(\frac{1}{2^{k}-2}, \frac{2^{k}-k-1}{2^{k}-2}\right)
$$

in which the term $N^{1-2^{2-k}} \lambda_{k}^{-1 /\left(2^{k}-2\right)}$ has no effect. However the situation with Theorem 11 is more complicated. None the less we are able to produce a series of new exponent pairs.

Before stating the result we remind the reader of the necessary background. Let $s$ and $c$ be positive constants, and let $\mathcal{F}(s, c)$ be the set of quadruples $(N, I, f, y)$ where $y \geq N^{s}$ are positive real numbers, $I$ is a subinterval of $(N, 2 N]$, and $f$ is an infinitely differentiable function on $I$, with

$$
\left|f^{(n+1)}(x)-\frac{d^{n}}{d x^{n}}\left(y x^{-s}\right)\right| \leq c\left|\frac{d^{n}}{d x^{n}}\left(y x^{-s}\right)\right|
$$

for $x \in I$, for all $n \geq 0$. We then say that $(p, q)$ is an exponent pair, if $p$ and $q$ lie in the range $0 \leq p \leq \frac{1}{2} \leq q \leq 1$, and for each $s$ there is a corresponding $c=c(p, q, s)>0$ such that

$$
\sum_{n \in I} e(f(n)) \ll_{p, q, s}\left(y N^{-s}\right)^{p} N^{q},
$$


uniformly for all quadruples $(N, I, f, y) \in \mathcal{F}(s, c)$.

We then have the following.

Theorem 2 For any integer $k \geq 3$ and any real $\varepsilon>0$ there is an exponent pair given by

and

$$
p=\frac{2}{(k-1)^{2}(k+2)},
$$

$$
q=\frac{k^{3}+k^{2}-5 k+2}{k(k-1)(k+2)}+\varepsilon=1-\frac{3 k-2}{k(k-1)(k+2)}+\varepsilon
$$

In fact we are able to handle a much weaker condition on $f$. Let $\mathcal{A}=\left(a_{k}\right)_{3}^{\infty}$ and $\mathcal{B}=\left(b_{k}\right)_{3}^{\infty}$ be sequences of positive real numbers, and let $\mathcal{G}(\mathcal{A}, \mathcal{B})$ be the set of quadruples $(N, I, g, T)$ where $T \geq N$ are positive real numbers, $I$ is a subinterval of $(N, 2 N]$, and $g$ is an infinitely differentiable function on $I$, with

$$
a_{k} T N^{-k} \leq\left|g^{(k)}(x)\right| \leq b_{k} T N^{-k}
$$

for $x \in I$, and for all $k \geq 3$. We then have the following.

Theorem 3 For any integer $k \geq 3$ and any real $\varepsilon>0$, let $p$ and $q$ be given by (5) and (6). Then

$$
\sum_{n \in I} e(g(n)) \ll_{k, \varepsilon, \mathcal{A}, \mathcal{B}}\left(T N^{-1}\right)^{p} N^{q},
$$

uniformly for $(N, I, g, T) \in \mathcal{G}(\mathcal{A}, \mathcal{B})$.

If $(N, I, g, y) \in \mathcal{F}\left(s, \frac{1}{4}\right)$, then $\left(N, I, g, y N^{1-s}\right) \in \mathcal{G}(\mathcal{A}, \mathcal{B})$ with

$$
a_{k}=\frac{3 \times 2^{1-2-k}}{4 s(s+1) \ldots(s+k-2)}, \quad b_{k}=\frac{5}{4 s(s+1) \ldots(s+k-2)} .
$$

The sequences $\mathcal{A}$ and $\mathcal{B}$ depend only on $s$, and we immediately see that Theorem 2 follows from Theorem 3 .

We next present a slightly weaker version of Theorem 3 which is somewhat more immediately intelligible. It will be convenient to write $T=N^{\tau}$.

Theorem 4 Let sequences $\mathcal{A}$ and $\mathcal{B}$, and a real number $\varepsilon>0$ be given, then

$$
\sum_{n \in I} e(g(n)) \ll_{\varepsilon, \mathcal{A}, \mathcal{B}} N^{1-49 /\left(80 \tau^{2}\right)+\varepsilon},
$$

uniformly for quadruples $(N, I, g, T) \in \mathcal{G}(\mathcal{A}, \mathcal{B})$ with $N \leq T^{1 / 2}$.

The constant $49 / 80$ arises from the use of an exponent pair

$$
\left(\frac{1}{20}, \frac{33}{40}\right)=A^{2} B A^{2} B(0,1)
$$

when $\tau=\frac{7}{2}$. One could improve the constant slightly by employing a better exponent pair. As will be clear from the proof, the constant $\frac{49}{80}$ may be replaced by $1-\delta$ for any small $\delta>0$, if we restrict to sufficiently large values $\tau \geq \tau(\delta)$.

As an example of Theorem 4 , if $t \geq 2$ we find that

$$
\sum_{n \in I} n^{-i t} \ll_{\varepsilon} N^{1-49 / 80 \tau^{2}+\varepsilon},
$$

for $\tau=(\log t) /(\log N) \geq 2$. This should be compared with (3). Using (7) we produce the following result. 
Theorem 5 Let $\kappa=\frac{8}{63} \sqrt{15}=0.4918 \ldots$. Then for any fixed $\varepsilon>0$ we have

$$
\zeta(\sigma+i t) \ll_{\varepsilon} t^{\kappa(1-\sigma)^{3 / 2}+\varepsilon}
$$

uniformly for $t \geq 1$ and $\frac{1}{2} \leq \sigma \leq 1$. Moreover we have

$$
\zeta(\sigma+i t) \ll_{\varepsilon} t^{\frac{1}{2}(1-\sigma)^{3 / 2}+\varepsilon}
$$

uniformly for $t \geq 1$ and $0 \leq \sigma \leq 1$.

One sees from the proof that $\kappa$ may be reduced to $2 / \sqrt{27}+\delta=0.3849 \ldots$ for any small $\delta>0$, if we restrict $\sigma$ to a suitably small range $\sigma(\delta) \leq \sigma \leq 1$. The corresponding result in the work of Ford [2, Theorem 1] states that

$$
|\zeta(\sigma+i t)| \leq 76.2 t^{4.45(1-\sigma)^{3 / 2}}(\log t)^{2 / 3}
$$

for $t \geq 3$ and $\frac{1}{2} \leq \sigma \leq 1$. Thus we have reduced the constant 4.45 to $0.4918 \ldots$. Unfortunately our result yields no useful information when $\sigma$ tends to 1 , which is a critical situation in many applications. Moreover we do not have the explicit order constant that Ford finds.

As Ford explains, there are a number of interesting corollaries, for which we merely have to replace the constant $B=4.45$ by $B=0.492$ in the arguments given in [2, Pages 566 and 567]. We can feed our bound into the zero-density theorem of Montgomery 4, Theorem 12.3] (with $1-\alpha=4.93(1-\sigma$ ) as used by Ford [2, Page 566]) to give the following.

Corollary 1 We have

$$
N(\sigma, T) \ll_{\varepsilon} T^{6.42(1-\sigma)^{3 / 2}+\varepsilon}
$$

for $\frac{9}{10} \leq \sigma \leq 1$.

For moments of the Riemann Zeta-function we have:

Corollary 2 For any positive integer $k$ one has

$$
\int_{0}^{T}|\zeta(\sigma+i t)|^{2 k} d t \sim T \sum_{1}^{\infty} d_{k}(n)^{2} n^{-2 \sigma}
$$

as $t \rightarrow \infty$, for any fixed $\sigma \geq 1-0.534 k^{-2 / 3}$.

For the generalized divisor problem we have:

Corollary 3 For any positive integer $k$ the error term $\Delta_{k}(x)$ in the generalized divisor problem satisfies

$$
\Delta(x) \ll_{k} x^{1-0.849 k^{-2 / 3}} .
$$

In Section 2 we will reduce the proof of Theorem 1 to a two-variable counting problem involving fractional parts of the derivatives $f^{(j)}(n)$. Section 3 shows how this counting problem is tackled, and finally Section 4 completes the proof of our theorems.

Acknowledgement. This work was supported by EPSRC grant number $\mathrm{EP} / \mathrm{K} 021132 \mathrm{X} / 1$ 


\section{Initial Steps}

Our goal in the first stage of the proof is to estimate the sum

$$
\Sigma=\sum_{n \leq N} e(f(n))
$$

in terms of $J_{s, l}(P)$, together with, a counting function involving the fractional parts of numbers of the form $f^{(j)}(n) / j$ !.

Lemma 1 Let $k \geq 2$ be an integer, and suppose that $f(x):[0, N] \rightarrow \mathbb{R}$ has continuous derivatives of order up to $k$ on $(0, N)$. Suppose further that

$$
0<\lambda_{k} \leq f^{(k)}(x) \leq A \lambda_{k}, \quad x \in(0, N)
$$

and that $A \lambda_{k} \leq \frac{1}{4}$. Then

$$
\Sigma \ll H+k^{2} N^{1-1 / s} \mathcal{N}^{1 / 2 s}\left\{H^{-2 s+k(k-1) / 2} J_{s, k-1}(H)\right\}^{1 / 2 s},
$$

where $H=\left[\left(A \lambda_{k}\right)^{-1 / k}\right]$ and

$$
\mathcal{N}=\#\left\{m, n \leq N:\left\|\frac{f^{(j)}(m)}{j !}-\frac{f^{(j)}(n)}{j !}\right\| \leq 2 H^{-j} \text { for } 1 \leq j \leq k-1\right\} .
$$

If $J_{s, k-1}(H) \ll_{\varepsilon, k} H^{2 s-k(k-1) / 2+\varepsilon}$ as in (4) the estimate in the lemma reduces to

$$
\Sigma \ll_{\varepsilon, k} H+N^{1-1 / s+\varepsilon} \mathcal{N}^{1 / 2 s} .
$$

Here we would want to choose $s$ to be as small as possible, and since we are taking $l=k-1$ this means that we will have $s=k(k-1) / 2$.

The lemma is clearly trivial if $H \geq N$, and we may therefore suppose for the proof that $H \leq N$. For any positive integer $H \leq N$ we will have

$$
H \Sigma=\sum_{h \leq H} \sum_{-h<n \leq N-h} e(f(n+h))=\sum_{h \leq H} \sum_{1 \leq n \leq N-H} e(f(n+h))+O\left(H^{2}\right),
$$

so that

$$
\Sigma=H^{-1} \sum_{n \leq N-H} \sum_{h \leq H} e(f(n+h))+O(H) .
$$

We proceed to approximate $f(n+h)$ by the polynomial

$$
f_{n}(h):=f(n)+f^{\prime}(n) h+\ldots+\frac{f^{(k-1)}(n)}{(k-1) !} h^{k-1}
$$

To do this we set $g_{n}(x)=f(n+x)-f_{n}(x)$ and use summation by parts to obtain the bound

$$
\sum_{h \leq H} e(f(n+h)) \ll\left|S_{n}(H)\right|+\int_{0}^{H}\left|S_{n}(x) g_{n}^{\prime}(x)\right| d x,
$$

where we have written

$$
S_{n}(x)=\sum_{h \leq x} e\left(f_{n}(h)\right)
$$


for convenience.

If $0 \leq x \leq H$ we may use Taylor's Theorem with Lagrange's form of the remainder to show that

$$
f^{\prime}(n+x)=f_{n}^{\prime}(x)+\frac{f^{(k)}(\xi)}{k !} x^{k-1}
$$

for some $\xi \in(n, n+x) \subseteq(0, N)$. It follows that

$$
g_{n}^{\prime}(x) \ll A \lambda_{k} H^{k-1}
$$

on $[0, H]$. With the choice $H=\left[\left(A \lambda_{k}\right)^{-1 / k}\right]$ we find that

$$
\sum_{h \leq H} e(f(n+h)) \ll\left|S_{n}(H)\right|+H^{-1} \int_{0}^{H}\left|S_{n}(x)\right| d x .
$$

The bound (11) now yields

$$
\Sigma \ll H+H^{-1} \sum_{n \leq N-H}\left|S_{n}(H)\right|+H^{-2} \int_{0}^{H}\left\{\sum_{n \leq N-H}\left|S_{n}(x)\right|\right\} d x .
$$

It then follows that there is a positive integer $H_{0} \leq H$ such that

$$
\Sigma \ll H+H^{-1} \sum_{n \leq N-H}\left|S_{n}\left(H_{0}\right)\right| .
$$

Now suppose that $\boldsymbol{\alpha} \in[0,1]^{k-1}$ and

$$
\left\|f^{(j)}(n) / j !-\alpha_{j}\right\| \leq H^{-j} \text { for } 1 \leq j \leq k-1
$$

where

$$
\|\theta\|=\min _{n \in \mathbb{Z}}|\theta-n|
$$

as usual. We proceed to replace $f_{n}(h)$ by

$$
f(h ; \boldsymbol{\alpha})=\alpha_{1} h+\ldots+\alpha_{k-1} h^{k-1}
$$

as follows. Firstly we remove the constant term $f(n)$ from $f_{n}(h)$. This has no effect on $\left|S_{n}\left(H_{0}\right)\right|$. Next, we replace each coefficient $f^{(j)}(n) / j$ ! by $c_{j}$, say, with $f^{(j)}(n) / j !-c_{j} \in \mathbb{Z}$, so that $\left|c_{j}-\alpha_{j}\right| \leq H^{-j}$, and denote the resulting polynomial by $f_{n}^{*}(h)$. If we write

$$
S_{n}^{*}\left(H_{0}\right)=\sum_{h \leq H_{0}} e\left(f_{n}^{*}(h)\right)
$$

then clearly $\left|S_{n}\left(H_{0}\right)\right|=\left|S_{n}^{*}\left(H_{0}\right)\right|$. Moreover

$$
\frac{d}{d x}\left(f(x ; \boldsymbol{\alpha})-f_{n}^{*}(x)\right) \ll k^{2} \max _{j \leq k-1}\left|c_{j}-\alpha_{j}\right| H^{j-1} \ll k^{2} H^{-1} .
$$

It therefore follows on summing by parts that

$$
S_{n}^{*}\left(H_{0}\right) \ll\left|S\left(H_{0} ; \boldsymbol{\alpha}\right)\right|+k^{2} H^{-1} \int_{0}^{H_{0}}|S(x ; \boldsymbol{\alpha})| d x,
$$


where we have set

$$
S(x ; \boldsymbol{\alpha})=\sum_{h \leq x} e(f(h ; \boldsymbol{\alpha})) .
$$

We may therefore conclude that

$$
S_{n}^{*}\left(H_{0}\right) \ll 2^{-k} H^{k(k-1) / 2}\left\{\int_{\boldsymbol{\alpha}}\left|S\left(H_{0} ; \boldsymbol{\alpha}\right)\right| d \boldsymbol{\alpha}+k^{2} H^{-1} \int_{0}^{H_{0}} \int_{\boldsymbol{\alpha}}|S(x ; \boldsymbol{\alpha})| d \boldsymbol{\alpha} d x\right\},
$$

where the integral over $\boldsymbol{\alpha}$ is for vectors in $[0,1]^{k-1}$ satisfying (13).

For each $\boldsymbol{\alpha} \in[0,1]^{k-1}$ we now define

$$
\nu(\boldsymbol{\alpha})=\#\left\{n \leq N-H:\left\|f^{(j)}(n) / j !-\alpha_{j}\right\| \leq H^{-j} \text { for } 1 \leq j \leq k-1\right\} .
$$

We then find that

$$
\sum_{n \leq N-H}\left|S_{n}\left(H_{0}\right)\right| \ll 2^{-k} H^{k(k-1) / 2}\left\{I\left(H_{0}\right)+k^{2} H^{-1} \int_{0}^{H_{0}} I(x) d x\right\},
$$

with

$$
I(x)=\int_{0}^{1} \ldots \int_{0}^{1}|S(x ; \boldsymbol{\alpha})| \nu(\boldsymbol{\alpha}) d \boldsymbol{\alpha} .
$$

We easily see that

$$
\int_{0}^{1} \cdots \int_{0}^{1} \nu(\boldsymbol{\alpha}) d \boldsymbol{\alpha}=2^{k-1} H^{-k(k-1) / 2}(N-H)
$$

and that

$$
\int_{0}^{1} \cdots \int_{0}^{1} \nu(\boldsymbol{\alpha})^{2} d \boldsymbol{\alpha} \leq 2^{k-1} H^{-k(k-1) / 2} \mathcal{N}
$$

where $\mathcal{N}$ is defined in Lemma 1 . Moreover

$$
\int_{0}^{1} \cdots \int_{0}^{1}|S(x ; \boldsymbol{\alpha})|^{2 s} d \boldsymbol{\alpha}=J_{s, k-1}(x)
$$

in the notation of (2). Since $J_{s, k-1}(P)$ is non-decreasing in $P$ this last integral may be bounded by $J_{s, k-1}(H)$.

Hence, by Hölder's inequality, for any positive integer $s$ we have

$$
I(x) \ll 2^{k} H^{-k(k-1) / 2} N^{1-1 / s} \mathcal{N}^{1 / 2 s}\left\{H^{k(k-1) / 2} J_{s, k-1}(H)\right\}^{1 / 2 s} .
$$

Thus (14) yields

$$
\sum_{n \leq N-H}\left|S_{n}\left(H_{0}\right)\right| \ll k^{2} N^{1-1 / s} \mathcal{N}^{1 / 2 s}\left\{H^{k(k-1) / 2} J_{s, k-1}(H)\right\}^{1 / 2 s}
$$

and (12) gives us

$$
\Sigma \ll H+k^{2} N^{1-1 / s} \mathcal{N}^{1 / 2 s}\left\{H^{-2 s+k(k-1) / 2} J_{s, k-1}(H)\right\}^{1 / 2 s}
$$

as required. 


\section{The counting function $\mathcal{N}$}

Naturally our next task is to bound $\mathcal{N}$. The original approach taken by Vinogradov, as described in Titchmarsh [8, Chapter 6], merely used an $L^{\infty}$ bound for $\nu(\boldsymbol{\alpha})$. One discards all the information on $f^{(j)}(n) / j$ ! for $j \leq k-2$ and uses only the case $j=k-1$. One then employs a standard procedure given by the following trivial variant of [8, Lemma 6.11], for example.

Lemma 2 Let $N$ be a positive integer, and suppose that $g(x):[0, N] \rightarrow \mathbb{R}$ has a continuous derivative on $(0, N)$. Suppose further that

$$
0<\mu \leq g^{\prime}(x) \leq A_{0} \mu, \quad x \in(0, N) .
$$

Then

$$
\#\{n \leq N:\|g(n)\| \leq \theta\} \ll\left(1+A_{0} \mu N\right)\left(1+\mu^{-1} \theta\right) .
$$

We fix $m$ and take

$$
g(x)=\frac{f^{(k-1)}(x)-f^{(k-1)}(m)}{(k-1) !}
$$

and $\mu=\lambda_{k} /(k-1) !, A_{0}=A$. This leads to a bound

$$
\mathcal{N} \ll(k-1) ! N\left(1+A N \lambda_{k}\right)\left(1+H^{1-k} \lambda_{k}^{-1}\right) .
$$

Under the assumption $A \lambda_{k} \leq \frac{1}{4}$ in Lemma 1 we have

$$
H^{1-k} \lambda_{k}^{-1} \asymp\left(A \lambda_{k}\right)^{1-1 / k} \lambda_{k}^{-1}=A\left(A \lambda_{k}\right)^{-1 / k} \geq A \geq 1,
$$

whence our bound produces

$$
\mathcal{N} \ll A^{2}(k-1) ! N \lambda_{k}^{-1 / k}\left(1+N \lambda_{k}\right) .
$$

If one inserts this into (10) with $s=k(k-1) / 2$ one gets an estimate

$$
\begin{array}{rll}
\Sigma & \ll_{\varepsilon, k} & \left(A \lambda_{k}\right)^{-1 / k}+N^{1-1 / s+\varepsilon}\left\{A^{2} N \lambda_{k}^{-1 / k}\left(1+N \lambda_{k}\right)\right\}^{1 / 2 s} \\
& \ll_{\varepsilon, k} & A N^{\varepsilon}\left\{\lambda_{k}^{-1 / k}+N^{1-1 / k(k-1)} \lambda_{k}^{-1 / k^{2}(k-1)}+N \lambda_{k}^{1 / k^{2}}\right\} .
\end{array}
$$

In fact the first term can be dropped, giving

$$
\Sigma \ll_{\varepsilon, k} A N^{\varepsilon}\left\{N \lambda_{k}^{1 / k^{2}}+N^{1-1 / k(k-1)} \lambda_{k}^{-1 / k^{2}(k-1)}\right\} .
$$

To see this we note that we have

$$
\Sigma \ll N^{1-1 / k(k-1)} \lambda_{k}^{-1 / k^{2}(k-1)}
$$

trivially unless

$$
N^{1-1 / k(k-1)} \lambda_{k}^{-1 / k^{2}(k-1)} \leq N .
$$

In this latter case however one sees that

$$
\lambda_{k}^{-1 / k} \leq N^{1-1 / k(k-1)} \lambda_{k}^{-1 / k^{2}(k-1)} .
$$

We may therefore regard (16) as being the result that Vinogradov's method achieves, given the results of Wooley [11] and Bourgain, Demeter and Guth [1. It is already a remarkable improvement on (1), replacing the critical exponent $1 /\left(2^{k}-2\right)$ by $1 / k^{2}$. Thus, in appropriate circumstances, we get an improvement as soon as $k \geq 5$. Our goal in this section is to make the following small further sharpening in the estimation of $\mathcal{N}$. 
Lemma 3 When $k \geq 3$ we have

$$
\mathcal{N} \ll((k-1) ! A)^{4}\left(N+\lambda_{k} N^{2}+\lambda_{k}^{-2 / k}\right) \log N .
$$

Apart from the term $\lambda_{k}^{-2 / k}$, which is insignificant in applications, this represents an improvement of (15) by a factor $\ll_{A, k} \lambda_{k}^{1 / k}$.

On the one hand our proof will use the fact that $\mathcal{N}$ is a counting function of two variables $m$ and $n$. On the other we shall use information about both $f^{(k-1)}$ and $f^{(k-2)}$. The reader may find it slightly surprising in the light of this that our bound depends on $\lambda_{k}$ only, and not on estimates for other derivatives $f^{(j)}$. The introduction of $\mathcal{N}$, and our procedure for estimating it, are the only really new aspects to this paper.

We begin our analysis by assuming that $k \geq 3$ and noting that $\mathcal{N}$ is at most

$$
\mathcal{N}_{1}=\#\left\{m, n \leq N:\left\|\frac{f^{(j)}(m)}{j !}-\frac{f^{(j)}(n)}{j !}\right\| \leq 2 H^{-j} \text { for } j=k-2, k-1\right\} .
$$

We proceed to show that it suffices to consider pairs $m, n$ of integers that are relatively close. It will be convenient to write $B=4 H^{2-k}$ and $C=4 H^{1-k}$ and to set

$$
g_{1}(x)=\frac{f^{(k-2)}(x)}{(k-2) !}, \quad g_{2}(x)=\frac{f^{(k-1)}(x)}{(k-1) !} .
$$

We also define the doubly-periodic function

$$
\phi(x, y)=\max \left(1-B^{-1}\|x\|, 0\right) \max \left(1-C^{-1}\|y\|, 0\right),
$$

so that

$$
\mathcal{N}_{1} \ll \sum_{m, n \leq N} \phi\left(g_{1}(m)-g_{1}(n), g_{2}(m)-g_{2}(n)\right) .
$$

The function $\phi(x, y)$ has an absolutely convergent Fourier series

$$
\phi(x, y)=\sum_{r, s \in \mathbb{Z}} c_{r, s} e(r x+s y)
$$

with non-negative coefficients

$$
c_{r, s}=B C\left(\frac{\sin (\pi r B) \sin (\pi s C)}{\pi^{2} r s B C}\right)^{2}
$$

Thus

$$
\begin{aligned}
\mathcal{N}_{1} & \ll \sum_{r, s \in \mathbb{Z}} c_{r, s} \sum_{m, n \leq N} e\left(r\left(g_{1}(m)-g_{1}(n)\right)+s\left(g_{2}(m)-g_{2}(n)\right)\right) \\
& =\sum_{r, s \in \mathbb{Z}} c_{r, s}\left|\sum_{n \leq N} e\left(r g_{1}(n)+s g_{2}(n)\right)\right|^{2} .
\end{aligned}
$$

Let $K$ be a positive integer parameter, to be chosen later. We proceed to partition the range $(0, N]$ into $K$ intervals $I_{i}=\left(a_{i}, b_{i}\right]$ for $i \leq K$, having integer 
endpoints, and length $b_{i}-a_{i} \leq 1+N / K$. An application of Cauchy's inequality then yields

$$
\begin{aligned}
\mathcal{N}_{1} & \ll K \sum_{i \leq K} \sum_{r, s \in \mathbb{Z}} c_{r, s}\left|\sum_{n \in I_{i}} e\left(r g_{1}(n)+s g_{2}(n)\right)\right|^{2} \\
& =K \sum_{i \leq K} \sum_{r, s \in \mathbb{Z}} c_{r, s} \sum_{m, n \in I_{i}} e\left(r\left(g_{1}(m)-g_{1}(n)\right)+s\left(g_{2}(m)-g_{2}(n)\right)\right) \\
& =K \sum_{i \leq K} \sum_{m, n \in I_{i}} \phi\left(g_{1}(m)-g_{1}(n), g_{2}(m)-g_{2}(n)\right) \\
& \leq K \sum_{\substack{m, n \leq N \\
|m-n| \leq 1+N / K}} \phi\left(g_{1}(m)-g_{1}(n), g_{2}(m)-g_{2}(n)\right) .
\end{aligned}
$$

We may therefore conclude that $\mathcal{N}_{1} \ll K \mathcal{N}_{2}$, where $\mathcal{N}_{2}$ counts pairs of integers $m, n \leq N$ with $|m-n| \leq 1+N / K$ for which

$$
\left\|\frac{f^{(j)}(m)}{j !}-\frac{f^{(j)}(n)}{j !}\right\| \leq 4 H^{-j} \text { for } j=k-2, k-1 .
$$

If $|m-n| \leq 1+N / K$ we will have

$$
\left|\frac{f^{(k-1)}(m)}{(k-1) !}-\frac{f^{(k-1)}(n)}{(k-1) !}\right| \leq \frac{|m-n|}{(k-1) !} \sup \left|f^{(k)}\right| \leq A \lambda_{k}(1+N / K),
$$

by the mean-value theorem. We will choose

$$
K=1+\left[4 A \lambda_{k} N\right],
$$

so that

$$
\left|\frac{f^{(k-1)}(m)}{(k-1) !}-\frac{f^{(k-1)}(n)}{(k-1) !}\right| \leq \frac{1}{2},
$$

in view of our assumption that $A \lambda_{k} \leq \frac{1}{4}$. Thus if

$$
\left\|\frac{f^{(k-1)}(m)}{(k-1) !}-\frac{f^{(k-1)}(n)}{(k-1) !}\right\| \leq 4 H^{1-k}
$$

we must have

$$
\left|\frac{f^{(k-1)}(m)}{(k-1) !}-\frac{f^{(k-1)}(n)}{(k-1) !}\right| \leq 4 H^{1-k} .
$$

However the mean-value theorem also tells us that

$$
\left|\frac{f^{(k-1)}(m)}{(k-1) !}-\frac{f^{(k-1)}(n)}{(k-1) !}\right| \geq \frac{|m-n|}{(k-1) !} \inf \left|f^{(k)}\right| \geq \lambda_{k} \frac{|m-n|}{(k-1) !} .
$$

We therefore conclude that

$$
|m-n| \leq \frac{4(k-1) !}{\lambda_{k} H^{k-1}}
$$

for any pair $m, n$ counted by $\mathcal{N}_{2}$. 
There are $N$ pairs $m=n$ counted by $\mathcal{N}_{2}$. We consider the remaining pairs with $m>n$, the alternative case producing the same estimates by symmetry. Then $m=n+d$ with $1 \leq d \leq D$, where

$$
D=\min \left(N,\left[\frac{4(k-1) !}{\lambda_{k} H^{k-1}}\right]\right) .
$$

For each available value of $d$ we estimate the number of corresponding integers $n$ via Lemma 2 , taking

$$
g(x)=\frac{f^{(k-2)}(x+d)-f^{(k-2)}(x)}{(k-2) !} .
$$

Then

$$
g^{\prime}(x)=\frac{f^{(k-1)}(x+d)-f^{(k-1)}(x)}{(k-2) !}
$$

so that

$$
d \frac{\lambda_{k}}{(k-2) !} \leq d \frac{\inf \left|f^{(k)}\right|}{(k-2) !} \leq g^{\prime}(x) \leq d \frac{\sup \left|f^{(k)}\right|}{(k-2) !} \leq d \frac{A \lambda_{k}}{(k-2) !}
$$

by the mean-value theorem. We therefore apply the lemma with $\mu=\lambda_{k} d /(k-2)$ ! and $A_{0}=A$. This shows that each $d \geq 1$ contributes

$$
\begin{aligned}
& \ll(k-2) !\left(1+A N \lambda_{k} d\right)\left(1+H^{2-k} \lambda_{k}^{-1} d^{-1}\right) \\
& \ll(k-2) !\left(1+A N \lambda_{k} D\right)\left(D+H^{2-k} \lambda_{k}^{-1}\right) d^{-1} \\
& \ll((k-1) !)^{3} A\left(1+N H^{1-k}\right) H^{2-k} \lambda_{k}^{-1} d^{-1} \\
& \ll((k-1) ! A)^{3}\left(1+N \lambda_{k}^{1-1 / k}\right) \lambda_{k}^{-2 / k} d^{-1} .
\end{aligned}
$$

Summing for $d \leq D$ we therefore find that

$$
\mathcal{N}_{2} \ll N+((k-1) ! A)^{3}\left(1+N \lambda_{k}^{1-1 / k}\right) \lambda_{k}^{-2 / k} \log D .
$$

Since $k \geq 3, \lambda_{k} \leq 1$ and $D \leq N$ this simplifies to give

$$
\mathcal{N}_{2} \ll((k-1) ! A)^{3}\left(N+\lambda_{k}^{-2 / k}\right) \log N
$$

whence

$$
\begin{aligned}
\mathcal{N} \leq & \mathcal{N}_{1} \ll K \mathcal{N}_{2} \ll\left(1+A \lambda_{k} N\right)((k-1) ! A)^{3}\left(N+\lambda_{k}^{-2 / k}\right) \log N \\
& \ll((k-1) ! A)^{4}\left(N+\lambda_{k} N^{2}+\lambda_{k}^{-2 / k}+N \lambda_{k}^{1-2 / k}\right) \log N .
\end{aligned}
$$

Since $k \geq 3$ and $\lambda_{k} \leq 1$ we have $N \lambda_{k}^{1-2 / k} \leq N$, and Lemma 3 follows.

\section{Proof of the Theorems}

If we insert Lemma 3 into Lemma 11, and use the bound (4) with the choices $l=k-1, s=k(k-1) / 2$, we see that

$$
\Sigma \ll_{A, k, \varepsilon} N^{\varepsilon}\left(\lambda_{k}^{-1 / k}+N^{1-1 / k(k-1)}+N \lambda_{k}^{1 / k(k-1)}+N^{1-2 / k(k-1)} \lambda_{k}^{-2 / k^{2}(k-1)}\right) .
$$


The term $\lambda_{k}^{-1 / k}$ may be omitted, since the resulting bound

$$
\Sigma \ll_{A, k, \varepsilon} N^{\varepsilon}\left(N^{1-1 / k(k-1)}+N \lambda_{k}^{1 / k(k-1)}+N^{1-2 / k(k-1)} \lambda_{k}^{-2 / k^{2}(k-1)}\right)
$$

holds trivially when $N \leq N^{1-2 / k(k-1)} \lambda_{k}^{-2 / k^{2}(k-1)}$, while

$$
\lambda_{k}^{-1 / k} \leq N^{1-2 / k(k-1)} \lambda_{k}^{-2 / k^{2}(k-1)}
$$

when $N \geq N^{1-2 / k(k-1)} \lambda_{k}^{-2 / k^{2}(k-1)}$. This suffices for Theorem 1,

We turn next to Theorem [3. Suppose that $(N, I, g, T) \in \mathcal{G}(\mathcal{A}, \mathcal{B})$, and let $I$ have end points $N_{0}$ and $N_{0}+N_{1}$, so that $N_{1} \leq N$. We apply Theorem 1 to the function $f(x)=g\left(N_{0}+x\right)$, taking $\lambda_{k}=a_{k} T N^{-k}$ and $A=b_{k} / a_{k}$. (Since $f^{(k)}$ is differentiable it is continuous, and hence it cannot change sign if $\left|f^{(k)}(x)\right| \geq a_{k} T N^{-k}>0$. Taking complex conjugates of our sum if necessary we may therefore assume that $f^{(k)}(x)$ is positive on $I$.) It follows that if $k \geq 3$ then

$$
\begin{aligned}
\sum_{n \in I} e(g(n)) & \\
\ll_{\varepsilon, k, \mathcal{A}, \mathcal{B}} & N^{1+\varepsilon}\left(\lambda_{k}^{1 / k(k-1)}+N^{-1 / k(k-1)}+N^{-2 / k(k-1)} \lambda_{k}^{-2 / k^{2}(k-1)}\right) \\
\ll_{\varepsilon, k, \mathcal{A}, \mathcal{B}} & N^{1+\varepsilon}\left(N^{-1 /(k-1)} T^{1 / k(k-1)}+N^{-1 / k(k-1)}+T^{-2 / k^{2}(k-1)}\right) .
\end{aligned}
$$

We use the above bound for

$$
\frac{(k-1)^{2}+1}{k} \leq \tau<\frac{k^{2}+1}{k+1}
$$

where we define $\tau$ by $T=N^{\tau}$. For this range of $\tau$ we find that

$$
\begin{aligned}
& \max \left(\frac{\tau-k}{k(k-1)}, \frac{-1}{k(k-1)}, \frac{-2 \tau}{k^{2}(k-1)}\right) \\
& =\left\{\begin{array}{cc}
-1 / k(k-1), & \left((k-1)^{2}+1\right) / k \leq \tau \leq k-1, \\
(\tau-k) / k(k-1), & k-1 \leq \tau<\left(k^{2}+1\right) /(k+1),
\end{array}\right. \\
& \leq A_{k} \tau+B_{k} \text {, }
\end{aligned}
$$

where the coefficients $A_{k}$ and $B_{k}$ are chosen so that

$$
A_{k} \frac{(k-1)^{2}+1}{k}+B_{k}=\frac{-1}{k(k-1)}
$$

and

$$
A_{k} \frac{k^{2}+1}{k+1}+B_{k}=\frac{\left(k^{2}+1\right) /(k+1)-k}{k(k-1)}=\frac{-1}{k(k+1)} .
$$

One then calculates that

$$
A_{k}=\frac{2}{(k-1)^{2}(k+2)} \quad \text { and } \quad B_{k}=-\frac{3 k^{2}-3 k+2}{k(k-1)^{2}(k+2)} .
$$

If we now define $\phi(\tau):[2, \infty) \rightarrow \mathbb{R}$ by taking $\phi(\tau)=A_{k} \tau+B_{k}$ on

$$
\left[\frac{(k-1)^{2}+1}{k}, \frac{k^{2}+1}{k+1}\right)
$$


for each integer $k \geq 3$, we conclude that

$$
\sum_{n \in I} e(g(n)) \ll_{\varepsilon, \tau_{0}, \mathcal{A}, \mathcal{B}} N^{1+\phi(\tau)+\varepsilon},
$$

uniformly for $2 \leq \tau \leq \tau_{0}$. The function $\phi$ is continuous, and since the coefficients $A_{k}$ are monotonic decreasing $\phi$ is also convex. It follows that $\phi(\tau) \leq A_{k} \tau+B_{k}$ for any $\tau \in[2, \infty)$ and any $k \geq 3$. Thus

$$
\sum_{n \in I} e(g(n)) \ll_{\varepsilon, \tau_{0}, \mathcal{A}, \mathcal{B}} N^{1+B_{k}+\varepsilon} T^{A_{k}}=\left(T N^{-1}\right)^{p} N^{q},
$$

with $p, q$ given by (5) and (6). As before, this is uniform in any finite range $2 \leq \tau \leq \tau_{0}$. However if we set $\tau_{0}=1+(1-q) / p$ then $\tau_{0}$ will depend on $\varepsilon$ and $k$ alone. Moreover, if $\tau \geq \tau_{0}$ then we trivially have

$$
\sum_{n \in I} e(g(n)) \ll N \leq\left(T N^{-1}\right)^{p} N^{q} .
$$

Finally, if $\tau \leq 2$ we use the well known exponent pair $\left(\frac{1}{6}, \frac{2}{3}\right)$ to show that

$$
\sum_{n \in I} e(g(n)) \ll T^{1 / 6} N^{1 / 2} .
$$

When $k \geq 3$ one easily verifies that $q \geq p+1 / 2$ and $p+q \geq 5 / 6$ for the values (5) and (6), whence $T^{1 / 6} N^{1 / 2} \leq T^{p} N^{q-p}$ for $N \geq T^{1 / 2}$. It then follows that

$$
\sum_{n \in I} e(g(n)) \ll T^{1 / 6} N^{1 / 2} \leq T^{p} N^{q-p}
$$

for the remaining range $1 \leq \tau \leq 2$. This completes the proof of Theorem 3 ,

We move now to the proof of Theorem 4 . Let $\tau_{0}=\sqrt{49 / 80 \varepsilon^{2}}$. Then if $\tau \geq \tau_{0}$ we will trivially have

$$
\sum_{n \in I} e(g(n)) \ll N \leq N^{1-49 / 80 \tau^{2}+\varepsilon} .
$$

When $\tau \leq \tau_{0}$ we begin by handling the range $\frac{13}{3} \leq \tau \leq \tau_{0}$, for which we claim that $\phi(\tau) \leq-49 / 80 \tau^{2}$. This will clearly suffice, in view of the estimate (18). Since $\phi(\tau)$ is piecewise linear, while the function $-49 / 80 \tau^{2}$ is convex, it suffices to verify that $\phi(\tau) \leq-49 / 80 \tau^{2}$ at each of the points $\tau=\left(k^{2}+1\right) /(k+1)$, for $k \geq 5$. This condition is equivalent to

$$
\frac{\left(k^{2}+1\right)^{2}}{k(k+1)^{3}} \geq \frac{49}{80}
$$

However the fraction on the right is increasing for $k \geq 5$, and takes the value $169 / 270>49 / 80$ at $k=5$.

When $\frac{7}{2} \leq \tau \leq \frac{13}{3}$ we will use the bounds

$$
\sum_{n \in I} e(g(n)) \ll_{\varepsilon} N^{1-1 / 20+\varepsilon}, \quad\left(\frac{7}{2} \leq \tau \leq 4\right)
$$


and

$$
\sum_{n \in I} e(g(n)) \ll_{\varepsilon} N^{1-(5-\tau) / 20+\varepsilon}, \quad\left(4 \leq \tau \leq \frac{13}{3}\right)
$$

which come from the case $k=5$ of (17). Note that the first of these is valid in the longer range $\frac{17}{5} \leq \tau \leq 4$, but we shall only use it when $\frac{7}{2} \leq \tau \leq 4$. We therefore need to verify that $-1 / 20 \leq-49 / 80 \tau^{2}$ for $\frac{7}{2} \leq \tau \leq 4$ and that $-(5-\tau) / 20 \leq-49 / 80 \tau^{2}$ for $4 \leq \tau \leq \frac{13}{3}$. This is routine, but we observe that we have equality at $\tau=\frac{7}{2}$.

We next consider the case in which $\frac{59}{22} \leq \tau \leq \frac{7}{2}$, for which we use the bound

$$
\sum_{n \in I} e(g(n)) \ll(T / N)^{1 / 20} N^{33 / 40}=N^{1+(2 \tau-9) / 40}
$$

corresponding to the exponent pair $\left(\frac{1}{20}, \frac{33}{40}\right)$. (This pair is $A^{2} B A^{2} B(0,1)$ in the usual notation, see Titchmarsh [8, $\S 5.20]$, for example.) Again, it is routine to check that

$$
\frac{2 \tau-9}{40} \leq-\frac{49}{80 \tau^{2}}, \quad\left(\frac{59}{22} \leq \tau \leq \frac{7}{2}\right)
$$

Finally we examine the range $2 \leq \tau \leq \frac{59}{22}$, and here we use the bound

$$
\sum_{n \in I} e(g(n)) \ll(T / N)^{1 / 9} N^{13 / 18}=N^{1+(2 \tau-7) / 18}
$$

corresponding to the exponent pair $\left(\frac{1}{9}, \frac{13}{18}\right)$. (This pair is $A B A^{2} B(0,1)$ in the usual notation, see Titchmarsh [8, §5.20], for example.) Another routine check shows that

$$
\frac{2 \tau-7}{18} \leq-\frac{49}{80 \tau^{2}}, \quad\left(2 \leq \tau \leq \frac{59}{22}\right),
$$

thereby completing the proof of Theorem 4

We turn now to Theorem [5. If $\tau \geq 2$ we may use (7) along with a partial summation to obtain

$$
\sum_{n \in J} n^{-\sigma-i t} \ll_{\varepsilon} N^{1-49 / 80 \tau^{2}-\sigma+\varepsilon} \leq t^{(1-\sigma) \tau^{-1}-\frac{49}{80} \tau^{-3}+\varepsilon / 2}
$$

for any $\sigma \in\left[\frac{1}{2}, 1\right]$, and for any interval $J \subseteq(N, 2 N]$. As a function of $\tau \in(0, \infty)$ the exponent of $t$ is maximal at

$$
\tau=\sqrt{\frac{147}{80(1-\sigma)}},
$$

whence

$$
\sum_{n \in J} n^{-\sigma-i t} \ll_{\varepsilon} t^{\kappa(1-\sigma)^{3 / 2}+\varepsilon / 2} .
$$

Using a dyadic subdivision of $(0, N]$ we therefore have

$$
\sum_{n \leq N} n^{-\sigma-i t} \ll_{\varepsilon} t^{\kappa(1-\sigma)^{3 / 2}+3 \varepsilon / 4}
$$


for any $N \leq t^{1 / 2}$. A further summation by parts then shows that

$$
\sum_{n \leq M} n^{-1+\sigma-i t} \ll_{\varepsilon} M^{2 \sigma-1} t^{\kappa(1-\sigma)^{3 / 2}+\varepsilon} \ll_{\varepsilon} t^{\sigma-\frac{1}{2}+\kappa(1-\sigma)^{3 / 2}+\varepsilon}
$$

for any $M \leq t^{1 / 2}$. The required bound (8) then follows from the approximate functional equation for $\zeta(s)$.

The bound (9) follows from (8) when $\frac{1}{2} \leq \sigma \leq 1$, since $\kappa<\frac{1}{2}$. For the remaining range we use the functional equation, which shows that

$$
\zeta(\sigma+i t) \ll t^{\frac{1}{2}-\sigma}|\zeta(1-\sigma+i t)| \ll_{\varepsilon} t^{\frac{1}{2}-\sigma+\frac{1}{2} \sigma^{3 / 2}+\varepsilon} .
$$

However one can readily verify that

$$
\frac{1}{2}-\sigma+\frac{\sigma^{3 / 2}}{2} \leq \frac{(1-\sigma)^{3 / 2}}{2}
$$

for $0 \leq \sigma \leq \frac{1}{2}$, which completes the proof of Theorem 5 .

\section{References}

[1] J. Bourgain, C. Demeter and L. Guth, Proof of the main conjecture in Vinogradov's mean value theorem for degrees higher than three, arXiv:1512.01565,

[2] K. Ford, Vinogradov's integral and bounds for the Riemann zeta function, Proc. London Math. Soc. (3), 85 (2002), no. 3, 565-633.

[3] N.M. Korobov, Estimates of trigonometric sums and their applications, Uspehi Mat. Nauk, 13 (1958) no. 4 (82), 185-192.

[4] H.L. Montgomery, Topics in multiplicative number theory, Lecture Notes in Mathematics 227, (Springer, Berlin, 1971).

[5] O. Robert, On van der Corputs $k$-th derivative test for exponential sums, Indag. Math. (N.S.), to appear.

[6] O. Robert and P. Sargos, A fourth derivative test for exponential sums, Compositio Math., 130 (2002), no. 3, 275-292.

[7] P. Sargos, An analog of van der Corput's $A^{4}$-process for exponential sums, Acta Arith., 110 (2003), no. 3, 219-231.

[8] E.C. Titchmarsh, The theory of the Riemann zeta-function, Second edition, (Clarendon Press, Oxford University Press, New York, 1986).

[9] I.M. Vinogradov, New Estimates for Weyl Sums, Doklady Nauk SSSR, 8 (1935), no. 5, 195-198.

[10] I.M. Vinogradov, A new estimate of the function $\zeta(1+i t)$, Izv. Akad. Nauk SSSR. Ser. Mat., 22 (1958), 161-164.

[11] T.D. Wooley, The cubic case of the main conjecture in Vinogradov's mean value theorem, arXiv:1401.3150. 
Mathematical Institute,

Radcliffe Observatory Quarter,

Woodstock Road,

Oxford

OX2 6GG

UK

rhb@maths.ox.ac.uk 